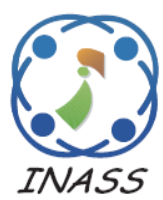

\title{
Color Enhancement Algorithm using the Integrated K-means Clustering and Inverted Otsu Method for Thermal Object Characterization
}

\author{
Chan Su Park ${ }^{1} \quad$ Hi Seok Kim ${ }^{1 *}$ \\ Cheongju University, South Korea \\ * Corresponding author's email: khs8391@cju.ac.kr
}

\begin{abstract}
Thermal imaging plays an important role in numerous applications like in defense, security, military, and other industry that requires a non-contact temperature measurement, etc. This proposed algorithm improves the thermal image especially during the thresholding process in which the desired object is separated from the background and can easily be identified. The separate extracted images produced by K-means clustering and inverted Otsu methods were processed by Canny edge detection and color mapping to highlight the necessary characteristics of the sampled thermal images. This work is synthesized with Xilinx Zync 7000 ZED ZC702. The experimental results of this blended combination of K-means and inverted Otsu methods show significant distinguishable features in terms of edge and color. It outperforms the other color correction method in terms of processing time. Moreover, this implementation reduced resource utilization, and minimizes the misclassified pixel in different noise variance.
\end{abstract}

Keywords: Thermal image, Inverted Otsu, K-means clustering, Object boundary, Color mapping.

\section{Introduction}

Thermal imaging is a technology used for analyzing the sample images which uses infrared detection process to identify the invisible infrared radiation effects, and making it visible to human eyes $[1,2]$. An ordinary camera is possible to record the data images event at a distance in real time. However, problems like the deterrent effects cannot be avoided like reflected lighting especially if regular RGB cameras were used [3]. The basic concepts in thermal imaging method are based on the relationship between the object's characteristics and the intensity of infrared radiation, which includes the temperature of objects and its surroundings. Thermal images have an endless number of applications in the industrial field and it is the most valuable diagnostic tool to detect any leak problems and can be used with thermal anomalies. Figure 1 shows an example of thermal imaging which helps to find and solve problems in process equipment. As shown in this Fig. 1 , over-heated pumps and compressors or any abnormal heat flow can be easily detected with thermal imaging.

Using a thermal imager equipment alone is very convenient, however, it still has many problems to be considered. It only defines the image preliminary characteristics Also, sample images might have unnecessary characteristics like with high noise, low

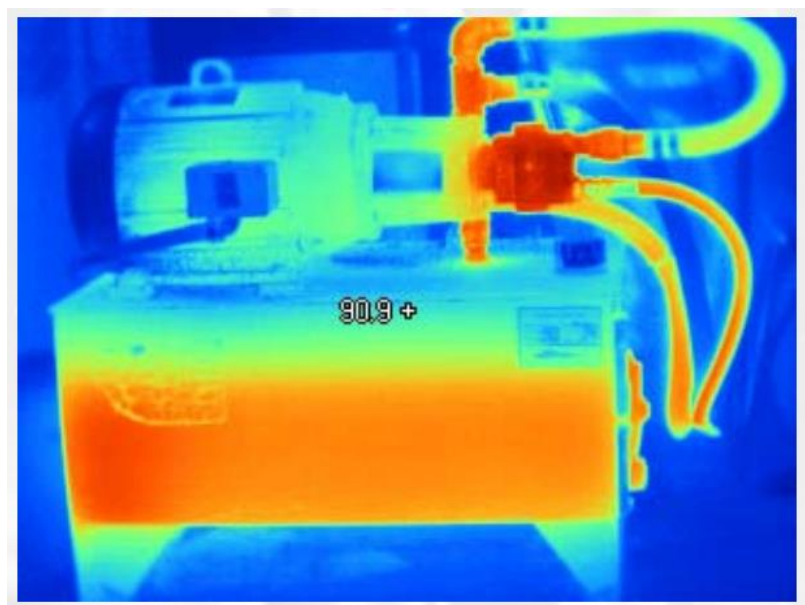

Figure. 1 Thermal imaging helps find/solve problems in process equipment with abnormal heat flow 
contrast, background interferences etc. [4]. Therefore an appropriate algorithm to facilitate these existing drawbacks including the thermal imager to enhance the quality of extracted thermal images. This paper shows an alternative algorithm to identify optimum thermal image characteristics and certain parameters related to thermal imaging and how to diagnose these faults.

This method is a combination of K-means clustering and inverted Otsu thresholding method as the basis of object and background separation. The integration concept aims to resolve the existing drawbacks of both methods. Although Otsu method is widely used for image thresholding. Ideally, an assumption on the histogram to have bimodal distribution and considered to possess a deep and pointed notch between two peaks. However, if the object area is smaller than the background area, resulting in a no modality in the histogram, [5]. Moreover, the gray level of the histogram is degraded if the variances of the object and the background intensities are larger than the mean difference, resulting to an incorrect threshold in the segmentation error of Otsu method. On the other hand, K-means clustering uses a local optimal method which does not require computing a gray level histogram before running and appropriate to multilevel thresholding than Otsu method. Also, it has certain limitations like difficult to predict the k-value, and it will not work properly in the global cluster. Moreover, with different initial partitions can result in different final clusters. And, it does not work well in clusters of different size and different density. This paper proposed a histogram clustering threshold based on color correction algorithm [6] by using an iterative threshold, minimum histogram difference is computed and then used to correct the target object. Achieving better thermal object characterizations in terms of shape information, edge images and an FPGA-based platform which eliminates unnecessary hardware and minimizes the processing time [7].

The rest of this paper is organized as follows. In Section 2, discussion of some related works to the proposed algorithm. Section 3 presents and describes the proposed algorithm, it includes implementation details. Experimental results and discussion are provided in Section 4. Finally, in Section 5, concludes this work.

\section{Review of related works}

Infrared thermography, thermal imaging, and thermal video are techniques under infrared imaging science. The range of radiation detection of thermal imaging cameras in the electromagnetic spectrum is around 9-14 $\mu \mathrm{m}$ and produce images called thermal image or thermograms. In analyzing a thermal image, a number of characteristics can be used to observe or, detect an object in a background. Normally, a detection process requires some necessary elements like target-to-background contrast, sensor performance, and cause by atmospheric attenuation [8 - 10].

In this section, presents some related works that are relevant to the proposed algorithm like the $\mathrm{K}$ means clustering scheme [11 - 13], Otsu method [14 - 16] and color mapping techniques [17 - 20] for image enhancement. Material investigations are also involved, which called as nondestructive testing techniques. It is defined as the method to examine a part, material, images, or system without impairing the original object [21, 22], resulting in high reliability and cost-effective method.

Also, thermal imagery is difficult to interpret and process with there is an existence of moisture absorption in the atmosphere. Normally, all objects emit infrared energy or electromagnetic energy at the speed of light as thermal heat. These electromagnetic energies are radiated in forms of waves with electrical and magnetic properties. Using a thermal imaging technology reveals that are not visible to the naked eye. Moreover, these thermal imageries can capture thermal images without making direct contact with the sample being tested. Therefore, the image being tested can capture thermal details at a safe distance and have a better chance of seeing temperature anomalies under normal operating conditions.

The thermal image is associated with different characteristics based on the IR radiation. These characteristics are absorptivity, emissivity, transmissivity, and reflectivity of infrared radiations [23] and the effects vary differently for every objects/material. Kirchhoff's law stated that the relationship between these characteristics as shown in Eq. (1)

$$
\eta+\varsigma+\xi=1
$$

where $\eta$ is the absorptivity, $\varsigma$ is reflectivity and $\xi$ is for transmissivity [24]. During thermal equilibrium of an object, the absorption is equal to emission. For opaque objects equation can be expressed as Eq. (2)

$$
\eta+\varsigma=1 \text { or } \psi+\varsigma=1
$$

where $\psi$ is the emissivity of the object.

Absorption of materials, thermal energy transmission, the intensity of emission, and the 


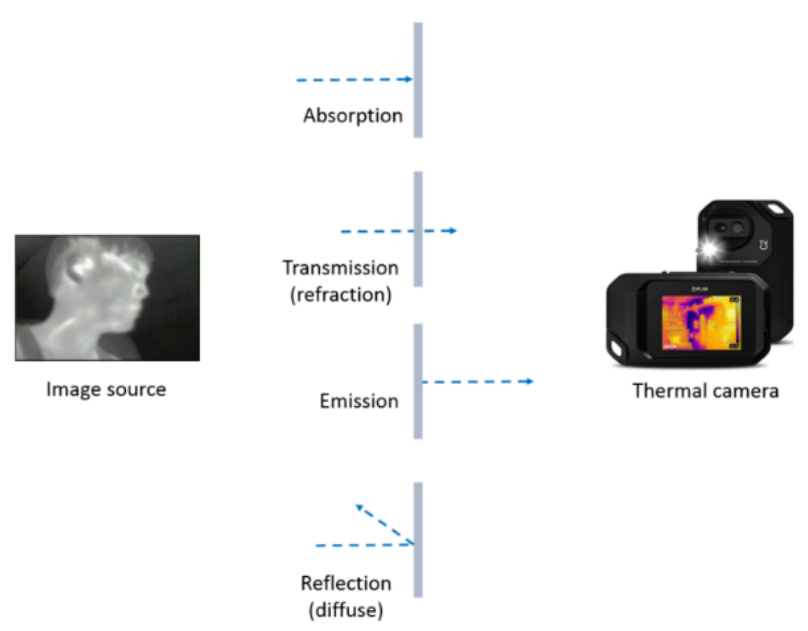

Figure. 2 Notations of the factors affecting the thermal properties of the sample image

reflection of radiation are some factors affecting the thermal properties of the sample object to be measured. Fig. 2 shows the factors that affect the sample object. The infrared sensors in thermal camera receive the total IR radiation emitted from the object's surface is directly related to the object's emissivity and its temperature, and define as the Stefan-Boltzmann Law [25-26] as shown in Eq. (3)

$$
E=\sigma \psi T^{4}
$$

where $E$ is the total amount of emitted radiation in an object per square meter, $\sigma$ is the Stefan-Boltzmann constant as $5.67 \times 10^{-8} \frac{\mathrm{W}}{\mathrm{m}^{2} \mathrm{~K}^{4}}$ and $T$ is designated for the temperature and measured in Kelvin $(K)$. Therefore, the object's temperature can determine if the emissivity of the object and total radiation emitted are known.

Digital image processing methods can improve the image information for further analysis and processing appropriate for data storage, transmission mode, and representation for automated machine recognition. Numerous algorithms are available were developed to enhance a more accurate image information [27-29] using digital image processing for different applications. Reference [30] describes one thermal image enhancement application using the inverted Otsu method. This work used an enhanced color mapping algorithm with inverted Otsu method however, it has no thresholding process for object segmentation and does not characterize the enhanced thermal image.

The thresholding algorithms presented in [31] proves that no single algorithm can enhance a thermal image. Also, the paper that is presented in [32] shows the selected thresholding methods from various categories and compared to measure the performances for different applications. However, no specific method was concluded which is appropriate for thermal image application. While in [33] presented a method for image thresholding and it was simulated in MATLAB. But it has no detailed description of the iteration process. The result in edge mapping which is presented in paper [34] uses edge direction and magnitude is far better against in using the edge magnitude alone.

Lastly, various papers presented with specific applications. In [35] discussed an algorithm for face detection utilizing thermal images, however, this paper has no actual hardware set-up and simulation. Reference [36] application is for thermal imaging of electrochemical power systems. This paper describes the related techniques of a thermal image for electrochemical power systems with the primary focus on fuel cells and batteries. Moreover, reference [37] is dedicated to analyze a thermal image in the medical field.

This proposed algorithm was compared with stationary wavelet transform and to the morphology technique. The stationary wavelet transform has been used in several images processing applications. The SWT transform is applied to the image for edge enhancement and this method does not decompose an image, the sub-bands will have the same size as the input image [38]. On the other hand, morphological image processing deals with the shape of features in an image [39]. The morphological operations are used to remove imperfections which were introduced during segmentation.

Moreover, there several characteristics of thermal images based on its applications. The three most important are listed below.

1. The response to the temperature of an object, as shown in equation (4), the radiance of an object is a function of its temperature. This equation describes the relationship is known as Planck's equation.

$$
W(\lambda, T)=e(\lambda) \frac{c_{1}}{\pi \lambda^{5}}\left[\exp \left(\frac{c_{2}}{\lambda T}\right)-1\right]
$$

where $W(\lambda, T)$ is the radiance for a wavelength $\lambda$ and measures in $\mu \mathrm{m}$ while $T$ stands for temperature in Kelvin, $e(\lambda)$ is the emissivity, $c_{1}$ and $c_{2}$ are the constants for this Planck's equation. The radiance is given in units of watts $\mathrm{m}^{-2}$ steradians $^{-1} \mu \mathrm{m}$ [40]. Therefore, a thermal imager (thermal camera) can be used as a means of measuring the object's temperature and can provide a two-dimensional distribution graph between the relative and actual temperature which is appropriate for different types of applications. 
2. The passive imaging is one of the important characteristics because there is no required external source of radiation is needed, the result provides a means of seeing at night-time, or even in poor illuminations.

3. Wavelength plays a vital part in visuality.

With these above characteristics, features like edges, shape, and size of the hottest regions can help to determine, characterize and detect the abnormalities of the subjected thermal images. Thus, this approach can play a significant role in many applications. Further enhancement and image processing can be employed to characterize the detected regions or segment for analyzations. Consequently, image processing plays a vital role in thermal imaging applications. Enhancing or developing an algorithm for thermal images can provide more reliable and accurate imaging method.

\section{Proposed algorithm}

This proposed algorithm aims to achieve a more vivid and sharp edge of thermal images for characterization. Figure 3 shows the proposed algorithm, the original input thermal image will be fed into three different channel (K-means clustering, inverted Otsu and high value thermal threshold processor), the resulting thermal images from $\mathrm{K}$ means clustering and inverted Otsu will be subjected to Canny edge detection to extract some useful information (characteristics) from different point of visibilities and minimize the amount of data (information) to be processed. Then, the red color mapping will extract the red portion from the output of the high-value thermal threshold. And all three outputs are combined together using a summing component.

To validate and verify the proposed algorithm, it was synthesized using the Xilinx Zynq 7000 ZC702 board. And, to manage the testing and simulation, an actual set-up was constructed to link with the Xilinx board. The set-up configuration is shown in Figure 4, it includes a thermal camera, FPGA board, and pc for configurations, etc. Also, Table 1 shows the details of the selected tools used for experimental testing. Additionally, different sample images were simulated using the three different techniques (stationary wavelet transform, the morphological technique, and the proposed algorithm) for comparison purposes.

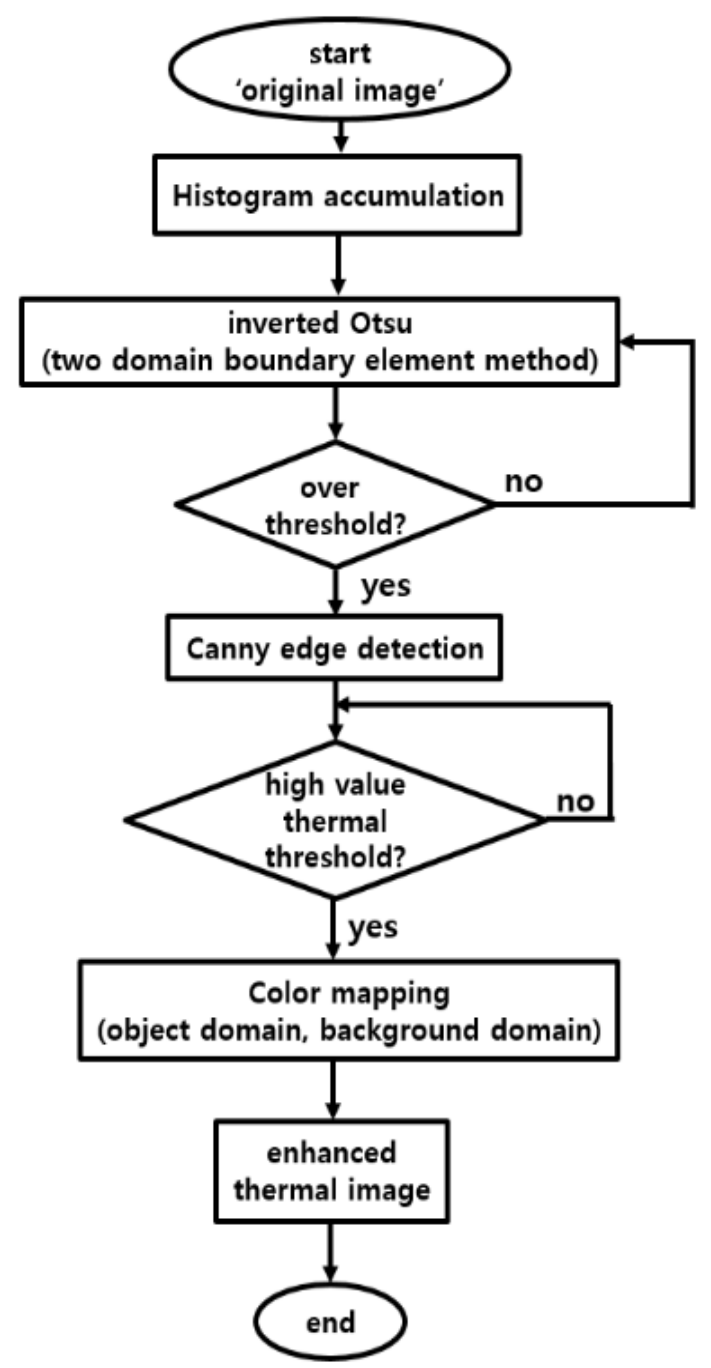

Figure. 3 Proposed algorithm

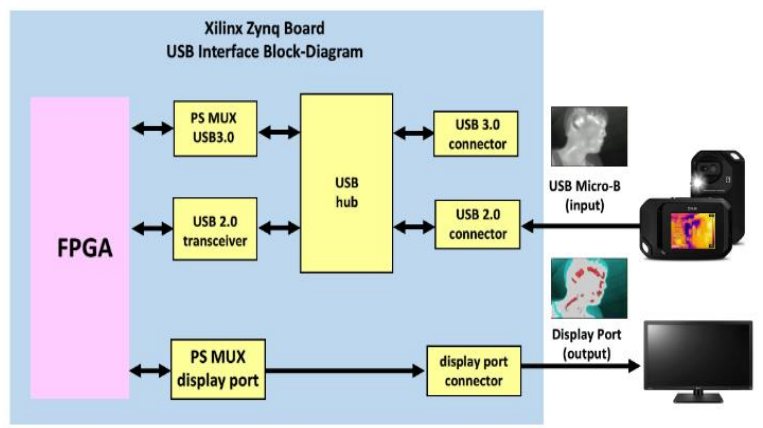

Figure. 4 Hardware set-up of the proposed algorithm

Table 1. Selected tools used for testing

\begin{tabular}{|c|c|}
\hline $\begin{array}{c}\text { Software Test } \\
\text { tool }\end{array}$ & Visual studio, openCV 2.4.11 \\
\hline $\begin{array}{c}\text { Hardware } \\
\text { test tool }\end{array}$ & $\begin{array}{c}\text { Vivado 2017.2 } \\
\text { (the complete design is a parallel } \\
\text { interface based on ARM and } \\
\text { FPGA) }\end{array}$ \\
\hline $\begin{array}{c}\text { Simulation } \\
\text { tool }\end{array}$ & Isim \\
\hline
\end{tabular}




\section{Discussion and analysis of results}

To evaluate the proposed algorithm, a comprehensive experimental analysis is conducted. The experimental set-up uses 20 different images which are analysed in the first instance to extract features and detect any noise. Only in case of discrepancy between the required ranges of the threshold, the misclassified pixels or frame is processed in order to enhance its quality. The image processing enhancement involves noise reduction which augments the signal to noise ratio, then to highlight some features which are not visible in the original images, contrast balance is used. Finally, edge detection method aims to define the discontinuities in the frame for consideration. Then, to illustrate the performance of the three different methods, the 20 different images were simulated using SWT, Morphology and the proposed algorithm.

The experimental results show that the output image using the proposed algorithm outperforms the other techniques in a number of important features. To assess the perceptual quality of the sample image, various features could be estimated that may represent subjective quality which characteristics could be considered as desired or unwanted. Table 2 shows the tabulated comparison between the three methods in terms of edge visibility, contrast, and brightness.

The results under the SWT method shows an ambiguity or almost unrecognizable to differentiate between the object and its background. While in the Morphology scheme, reveals partially visible the edge and contrast, but the object brightness is hazy. However, if this proposed algorithm is implemented the three thermal object characteristics are all noticeable. It shows that the enhanced thermal image by the applied algorithm has a more visible appearance and thermal object characteristics are more visually recognizable. The enhanced thermal image by the applied algorithm has a more visible appearance and thermal object characteristics are more visually recognizable. Compared to the other methods, the image enhanced by the algorithm maintains a better contrast, displays a higher brightness and colors to make a distinction between the object and its background.

The results show in Figure 5 are the thermal images tested during simulation of the proposed algorithm. These images provide a sharper edges making it more readily recognizable and show the outlines and boundaries between the object and its background. Relative to this, the resulting image emphasizes the thermal regions of the object as shown by the red area without losing its visibility. To further justify the performance of the proposed method under noisy, certain thermal images are contaminated by Gaussian noise and subjected to comparison using the three methods (SWT, Morphology, and proposed algorithm).

Table 2. Comparison of thermal object characteristics results using the three methods

\begin{tabular}{|c|c|c|c|}
\hline \multirow{2}{*}{$\begin{array}{c}\text { Thermal object } \\
\text { characteristics }\end{array}$} & SWT & Morphology & Proposed \\
\cline { 2 - 4 } & partially visible & partially visible & detectable \\
\hline edge visibility & $\begin{array}{c}\text { Ambiguous } \\
\text { (no difference) }\end{array}$ & partially distinct & distinct \\
\hline object to background contrast & almost dull & vague & noticeable \\
\hline object brightness &
\end{tabular}

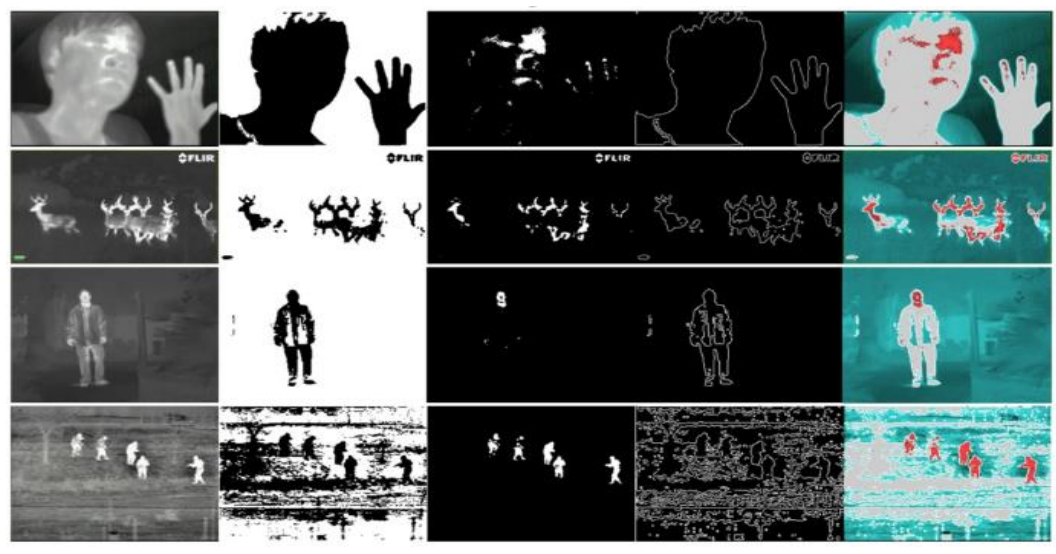

(a)

(b)

(c)

(d)

(e)

Figure. 5 (a) Original image, (b) integrated k-means clustering and inverted Otsu thresholding image, (c) heat source image, (d) Canny edge detection image, and (e) image results. 
Misclassified pixels

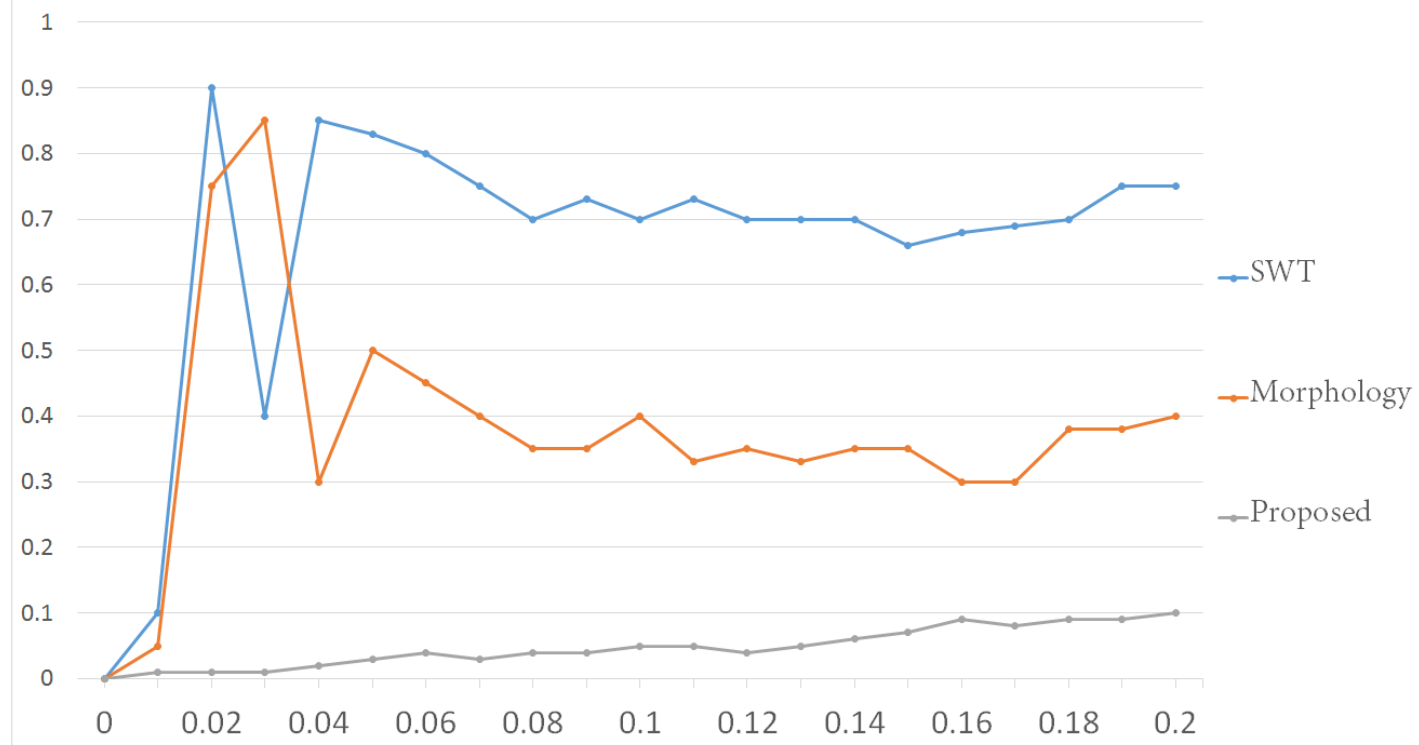

Noise variance

Figure. 6 Comparison of thermal images corrupted by Gaussian noise in different noise variances using the three methods (SWT, Morphology, and proposed algorithm)

Table 3. Processing time using the three methods

\begin{tabular}{|c|c|c|}
\hline Methods & $\begin{array}{c}\text { Software } \\
\text { CPU (i7 GHz), RAM (2,400 MHz) }\end{array}$ & $\begin{array}{c}\text { Hardware } \\
\text { Simulation clock Frequency: } 500 \mathrm{MHz}\end{array}$ \\
\hline SWT & $166 \mathrm{~ms}$ & $0.456 \mathrm{~ms}$ \\
\hline Morphology & $172 \mathrm{~ms}$ & $0.288 \mathrm{~ms}$ \\
\hline Proposed & $158 \mathrm{~ms}$ & $0.322 \mathrm{~ms}$ \\
\hline
\end{tabular}

Table 4. Resource utilization of SWT, Morphology, and Proposed Scheme

\begin{tabular}{|c|c|c|c|c|}
\hline Logic & SWT & Morphology & Proposed & Available \\
\hline Number of slice & 582 & 416 & 418 & 19,200 \\
\hline Number of 4 input LUTs & 388 & 400 & 384 & 19,200 \\
\hline Number of fully used LUT-FF pairs & 402 & 622 & 352 & 44,977 \\
\hline Number of bonded IOBs & 24 & 30 & 28 & 220 \\
\hline Number of Block RAM/FIFO & 2 & 2 & 2 & 32 \\
\hline
\end{tabular}

Fig. 6 shows the thermal images corrupted by Gaussian noise in different noise variances. Each noise variance tests 20 images. Since the noise variance is a random process, the testing repeats the process in 10 times and average the results.

Table 3 shows the operating time of the three methods simulated to verify the three methods. Table 4 shows the resource utilization using this FPGAbased implementation, it shows that the designed memory architecture is lower than the two other methods.

\section{Conclusion}

The proposed algorithm aims the targets especially in edge and boundary segmentation between the object and its background. The image results after the proposed method is more robust to noise, it minimizes the misclassified pixels especially when contaminated in Gaussian noise variance. Also, the thermal regions are naturally difficult to identify and almost invisible to the human eyes but using this proposed algorithm, it can easily be detected in the enhanced image result. Moreover, the presented results show a clear improvement on processing time and reduce on hardware utilization compared with SWT and Morphology technique.

In addition, this paper contributed to the development of in thermal images in visual recognition category and can be applied in healthcare, image retrieval, and data mining, likewise in industrial and personal robotics, manufacturing, scientific image analysis, and for surveillance and security applications. 


\section{References}

[1] X. Meng, "Applications and Research of Infrared Thermal Imaging Hazard Identification Technology", In: Proc. of the 2011 International Conference on Materials for Renewable Energy and Environment, pp. 1912-1916, 2011.

[2] R. Gade, T. Moeslund, S. Z. Nielsen, H. SkovPeterson, H. J. Andersen, K. Basselbjerg, H. T. Dam, O. Jensen, A. Jorgensen, H. Lahrmann, T. Madsen, E. S. Bala, and B. Povey, "Thermal Imaging Systems for Real-Time Applications in Smart Cities", International Journal of Computer Applications in Technology, Vol. 53, No. 4, pp. 290-308, 2016.

[3] F. Endres, J. Hess, and J. Sturm, "3-D Mapping with an RGB-D Camera", IEEE Transactions on Robotics, Vol. 30, No. 1, pp. 177-187, 2014.

[4] Z. Zhou, E. Malone, G. Sato dos Santos, N. Li, H. $\mathrm{Xu}$, and D. Holder, "Comparison of Different Quadratic Regularization for Electrical Impedance Tomography," In: Proc. of the $6^{\text {th }}$ Conference of the International Federation for Medical and Biological Engineering, pp. 200203, 2015.

[5] S. U. Lee, S. Y. Chung, and R. H. Park, "A Comparative Performance Study of Several Global Thresholding Techniques for Segmentation", Computer Vision, Graphics, and Image Processing, Vol. 52, No. 2, pp. 171-190, 1990.

[6] C. S. Park, H. S. Kim, and H W. Cha, "Histogram Color Correction for Multi-View Cording", In: Proc. of the 2014 International SoC Design Conference, pp. 252-253, 2014.

[7] R. S. Juan and H. S. Kim, "Reconfiguration of an FPGA-base Time-Triggered FlexRay Network Controller using EEDC", Journal of Circuits, Systems, and Computers, Vol. 27, No. 6, pp. 111, 2017.

[8] F. Fuliful, "Water Vapor Effect on 3-5 $\mu \mathrm{m}$ Band Thermal Imaging in Karbala City", Journal of Kerbala University, Vol. 12, No. 1, pp. 184-191, 2014.

[9] P. Jacobs, "Thermal Infrared Characterization of Ground Targets and Backgrounds", $2^{\text {nd }}$ Edition, SPIE Press Book, 2006.

[10] M. Ball and H. Pinkerton, "Factors Affecting the Accuracy of Thermal imaging Cameras in Volcanology", Journal of Geophysical Research, Vol. 111, No. 11, pp. 1-14, 2006.

[11] M. Mohd, S. Hernan, and Z. Sharif, "Application of K-means clustering in Hot Spot Detection for Thermal Infrared Images", In: Proc. of the 2017
IEEE Symposium on Computer Applications and Industrial Electronics, pp. 107-110, 2017.

[12] M. Etehadtayakol, S. Sadri, and E. Y. Ng, "Application of $\mathrm{K}$ and Fuzzy C-means for color segmentation of thermal infrared breast images", Journal of Medical Systems, Vol 34, No. 1, pp. $35-42,2010$.

[13] H. Zhou, Y. C. Soh, and X. Wu, "Integrated analysis of CFD Data with K-means Clustering Algorithm and Extreme Learning Machine for Localized HVAC Control", Applied Thermal Engineering, Vol. 76, pp. 98-104, 2015.

[14] S. Zhou, P. Yang, and W. Xie, "Infrared Image Segmentation Based on Otsu and Genetic Algorithm", In: Proc. of the 2011 International Conference on Multimedia Technology, Hangzhou, China, pp. 5421-5424, 2011.

[15] R. Heriansyah and S. Abu-Bakar, "Defect Detection in Thermal Image using Thresholding Technique", In: Proc. of the $6^{\text {th }}$ WSEAS International Conference on Circuits, Systems, Electronics, Control and Signal Processing, pp. 341-346, 2017.

[16] H. Vala and A. Baxi, "A Review on Otsu Image Segmentation Algorithm”, International Journal of Advanced Research in Computer Engineering and Technology, Vol. 2, No. 2, pp. 387-389, 2013.

[17] C. Tzeng, Z. Yang, and W. Tsai, "Adaptive Data Hiding in Palette Images by Color Ordering and Mapping with Security Protection", IEEE Transactions on Communications, Vol. 54, No. 5, pp. 791-800, 2004.

[18] H. S. Faridul, T. Pouli, and C. Chamaret, "A Survey of Color Mapping and its Applications," In: Proc. of Eurographics 2014, pp. 1-25, 2014.

[19] A. Toet and J. Walraven, "New false color mapping for image fusion", Optical Engineering, pp. 650-658, 1996.

[20] M. Hogervirst and A. Toet, "Improved Color Mapping Methods for Multiband Nighttime Image Fusion", Journal of Imaging, Vol. 3, No. 3, pp. 1-25, 2017.

[21] S. Kumar and D. Mahto, "Recent Trends in Industrial and Other Engineering Applications of Non-Destructive Testing: A Review," International Journal of Scientific and Engineering Research, Vol. 4, No. 9, pp. 183195, 2013.

[22] G. Dua and R. Mulaveesala, "Aperiodic Thermal Wave Imaging Approach for Non-Destructive Testing and Evaluation of Steel Material: A Numerical Study", Journal of Nanoengineering 
and Nanomanufacturing, Vol. 6, No. 4, pp. 265269, 2016.

[23] N. Dey, A. Ashour, and A. Althoupety, "Thermal Imaging in Medical Science", Recent Advances in Applied Thermal Imaging for Industrial Applications, pp. 1-20, 2017.

[24] P. M. Robitaille, "On the Validity of Kirchhoff's Law of Thermal Emission", IEEE Transmissions on Plasma Science, Vol. 31, No. 6, pp. 12631267, 2003.

[25] H. Paul, D. M. Greenberger, S.T. Stenholm, and W. P. Schleich, "The Stefan-Boltzmann Law: Two Classical Laws Give a Quantum One", Physica Scripta, pp. 1-7, 2015.

[26] E. Narimanov and I. Smolyaninov, "Beyond Stefan-Boltzmann Law: Thermal HyperConductivity”, CLEO Technical Digest, pp. 1-2, 2012.

[27] A. Kumar and V. Santhi, "A Review on Geometric Invariant Digital Image Watermarking Techniques", International Journal of Computer Applications, Vol. 12, No. 9, pp. 31-36, 2011.

[28] A. Ashour, S. Samanta, N. Dey, N. Kausar, W. B. Abdessalemkaraa, and A. E. Hassanien, "Computed Tomography Image Enhancement Using Cuckoo Search: A Log Transform Based Approach", Journal of Signal and Information Processing, Vol.6, No.3, pp. 244-257, 2015.

[29] A. Ghosh, A. Sarkar, A. Ashour, D. Blas-Timar, N. Dey, and V. Blas, "Grid Color Moment Features in Glaucoma Classification", International Journal of Advanced Computer Science and Applications, Vol. 6, No. 9, pp. 99107, 2015.

[30] B. H. Ko and H. S. Kim, "Using Enhanced-Color Mapping Algorithm for Object Boundary Segmentation", International Journal of Applied Engineering Research, Vol 12, No. 15, pp. 51875190, 2017.

[31] G. Leedham, C. Tan, K. Takru, J. H. N. Tan, and L. Mian, "Comparison of Some Thresholding Algorithms for Text/Background Segmentation in Difficult Documents Images", In: Proc. of the Seventh International Conference on Document Analysis and Recognition, pp. 1-6, 2003.

[32] M. Sezgin and B. Sankur, "Survey Over Image Thresholding Techniques and Quantitative Performance evaluation", Journal of Electronic Imaging, Vol. 13, No. 1, pp. 46-164, 2004.

[33] P. P. Vijay and N. C. Patil, "Gray Scale Image Segmentation using Otsu thresholding Optimal
Approach", Journal for Research, Vol. 2, No. 2, pp. 20-24, 2016.

[34] Q, Zhou, Z. Li, and J. K. Aggarwal, "Boundary Extraction in Thermal Images by Edge Map", In: Proc. of the 2004 ACM Symposium on Applied Computing, pp. 254-258, 2004.

[35] Y. K. Cheong, V. V. Yap, and H. Nisar, "A Novel Face Detection Algorithm using Thermal Imaging," In: Proc. of IEEE 2014 Symposium on Computer Applications and Industrial Electronics, pp. 208-213, 2014.

[36] J. Robinson, P. Shearing, and D. Brett, "Thermal Imaging of Electrochemical Power Systems: A Review", Journal of Imaging, Vol. 2, No. 1, pp. 1-20, 2016.

[37] E. F. Ring and K. Ammer, "Infrared Thermal Imaging in Medicine", Physiological Measurement Journal, Vol. 38, pp. 33-46, 2017.

[38] H. Demirel and G. Anbarjafari, "Image Resolution Enhancement by using Discrete and Stationary Wavelet Decomposition," IEEE Transactions on Image Processing, Vol. 20, No. 5, pp. 1458-1460, 2011.

[39] M. Goyal, "Morphological Image Processing", International Journal of Computer Science and Technology, Vol. 2, No. 4, pp. 161-165, 2011.

[40] T. Williams, "Thermal Imaging Cameras, Characteristics and Performance", CRC Press Taylor and Francis Group, 2009. 